\title{
A distributed activation energy model for clumped isotope bond reordering in carbonates
}

\author{
JORDON D HEMINGWAY ${ }^{1,2}$ AND GREGORY HENKES ${ }^{3}$ \\ ${ }^{1}$ Harvard University \\ ${ }^{2}$ ETH Zurich \\ ${ }^{3}$ Stony Brook University \\ Presenting Author: jordon_hemingway@fas.harvard.edu
}

Carbonate clumped isotopes $(\Delta 47)$ have become a widely applied method for paleothermometry, with applications spanning many environmental settings over hundreds of millions of years. However, $\Delta 47$-based paleothermometry can be complicated by closure temperature-like behavior whereby $\mathrm{C}-\mathrm{O}$ bonds are reset at elevated diagenetic or metamorphic temperatures, sometimes without obvious mineral alteration. Laboratory studies have constrained this phenomenon by heating well-characterized materials at various temperatures, observing temporal $\Delta 47$ evolution, and fitting results to kinetic models with prescribed $\mathrm{C}-\mathrm{O}$ bond reordering mechanisms. While informative, these models are inflexible regarding the nature of isotope exchange, leading to potential uncertainties when extrapolated to geologic timescales. Here, we instead propose that reordering kinetics arise naturally from random-walk 180 diffusion through the carbonate lattice, and we develop a "disordered" kinetic framework that treats $\mathrm{C}-\mathrm{O}$ bond reordering as a continuum of first-order processes occurring in parallel at different rates. We show theoretically that all previous models are specific cases of disordered kinetics; thus, our approach reconciles the transient defect/equilibrium defect and paired reaction-diffusion models. We estimate the rate coefficient distributions from published heating experiment data by finding a regularized inverse solution that best fits each $\Delta 47$ timeseries. Importantly, this approach does not assume a particular mechanism or energy distribution a priori. Resulting distributions are well-approximated as lognormal for all experiments on calcite or dolomite; aragonite experiments require more complex distributions that are consistent with a change in oxygen bonding environment during the transition to calcite. Presuming lognormal rate coefficient distributions and Arrhenius-like temperature dependence yields an underlying activation energy, $\mathrm{E}$, distribution that is Gaussian with a mean value of $\mu \mathrm{E}=$ $224.3 \pm 27.6 \mathrm{kJmol}-1$ and a standard deviation of $\sigma \mathrm{E}=17.4 \pm 0.7$ kJmol-1 $( \pm 1 \sigma$ uncertainty; $\mathrm{n}=24)$ for calcite and $\mu \mathrm{E}=$ $230.3 \pm 47.7 \mathrm{kJmol}-1$ and $\sigma \mathrm{E}=14.8 \pm 2.2 \mathrm{kJmol}-1 \quad(\mathrm{n}=4)$ for dolomite. These model results are adaptable to other minerals and may provide a basis for future experiments whereby the nature of carbonate $\mathrm{C}-\mathrm{O}$ bonds is altered (e.g., by inducing mechanical strain or cation substitution). Finally, we apply our results to geologically relevant heating/cooling histories and suggest that previous models underestimate low-temperature alteration but overestimate $\Delta 47$ blocking temperatures. 